\title{
CMS Outer Tracker: Operational Experience, Performance and Lessons Learned
}

\author{
T. Susa on the behalf of CMS Collaboration* \\ Rudjer Boskovic Institute \\ E-mail: tatjana.susa@irb.hr
}

The CMS Tracker consists of two tracking devices utilizing advanced silicon technology: the inner tracker with pixel detectors and the outer tracker composed of strip detectors. The outer tracker with more than 15000 silicon modules and $200 \mathrm{~m}^{2}$ of active silicon area is in its tenth year of operation at the LHC. We present the performance of the detector in the LHC Run 2 data taking. Results for signal-to-noise ratio, hit efficiency and single hit resolution will be presented. We review the behavior of the system when running at beyond-design instantaneous luminosity and describe challenges observed under these conditions. The evolution of detector operation parameters under the LHC conditions will also be discussed.

The 28th International Workshop on Vertex Detectors - Vertex2019

13-18 October, 2019

Lopud, Croatia

${ }^{*}$ Speaker. 


\section{Introduction}

The CMS tracker detector [1] is the central tracking device of the CMS experiment [2] at CERN Large Hadron Collider (LHC). The tracker is based on silicon sensor technology, utilizing pixels in the inner and microstrips in the outer part. In this contribution, operational experience and the performance of the outer strip detector will be presented.

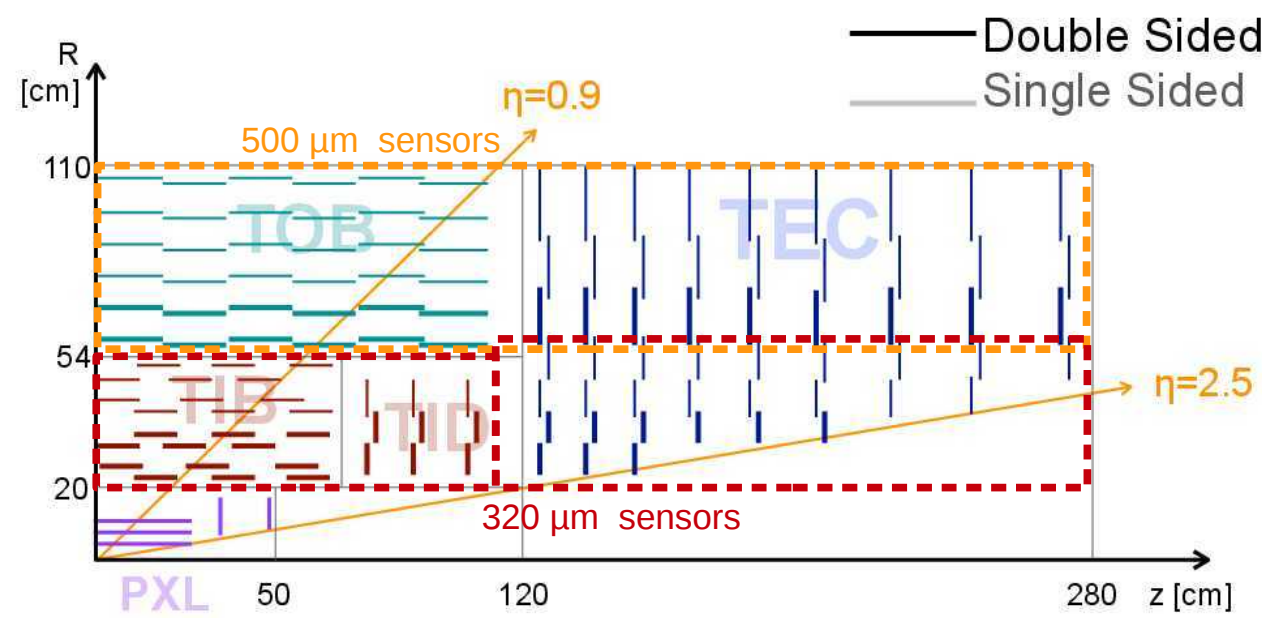

Figure 1: Quarter of the $r z$ slice of the CMS Silicon Strip tracker. Tracker Barrel and End-Cap layers and rings drawn by thin lines contain single-sided modules, those drawn by thick lines contain stereo modules. Modules in the TIB, TID and the first four rings of the TEC have $320 \mu \mathrm{m}$ thick silicon sensors, while in the TOB and rings 5-7 of the TEC $500 \mu \mathrm{m}$ thick silicon is used.

The CMS Silicon Strip Tracker (SST) consists of 15148 modules, arranged into four different sub-detectors, as shown in Fig. 1. In the Tracker Inner and Outer Barrels (TIB and TOB), modules are positioned in four and six layers around the z-axis, respectively. In the Tracker Inner Disks (TID) and the Tracker End-Caps (TEC), modules are arranged in rings with increasing radii and are mounted orthogonal to the z-axis on three and nine disks, respectively. Four layers in the barrel and multiple rings in the end-cap regions of the Silicon Strip tracker are equipped with stereo modules allowing for 2D measurement. These modules have two silicon sensors mounted back-to-back with their strips aligned at a $100 \mathrm{mrad}$ relative angle.

The silicon sensors are made up of single-sided p+ implants in the n-type silicon bulk with two different thicknesses: $320 \mu \mathrm{m}$ in the TIB, TID and inner TEC rings and $500 \mu \mathrm{m}$ in TOB and outer TEC rings.

\section{Performance of the CMS Outer Tracker during Run 2}

The fraction of inactive readout channels for the CMS Silicon Strip detector as a function of the delivered LHC integrated luminosity in the LHC Run 2 is presented in Fig. 2. Sources of inactive components include: non-functional read-out channels excluded from the cabling (typically Front End Drivers), unpowered groups of modules and single APV25 readout chips or groups of strips masked from the offline reconstruction by a Prompt Calibration loop algorithm [3], which identifies 


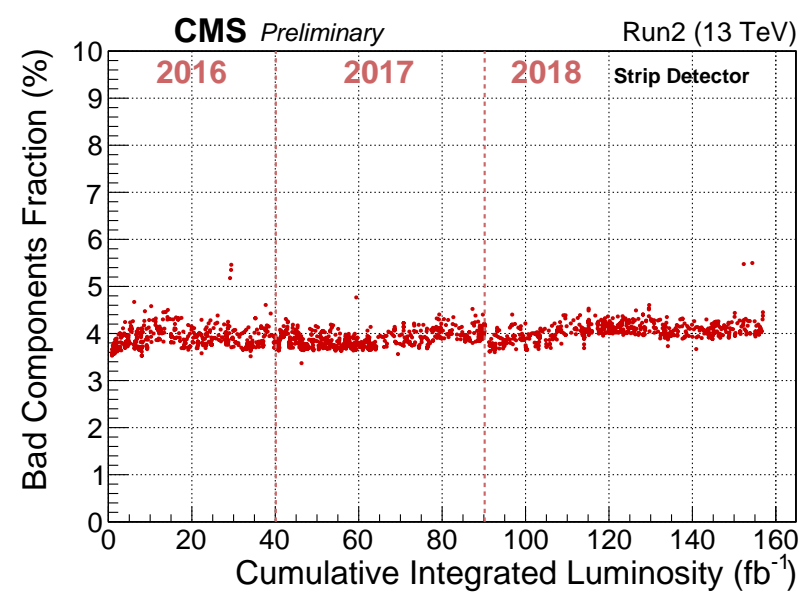

Figure 2: The fraction of inactive components for the CMS silicon strip detector as a function of the delivered LHC integrated luminosity.

very noisy channels based on the median occupancy. The fraction of the inactive components was very stable during LHC Run 2 and amounts to about $4 \%$ of the total number of channels.

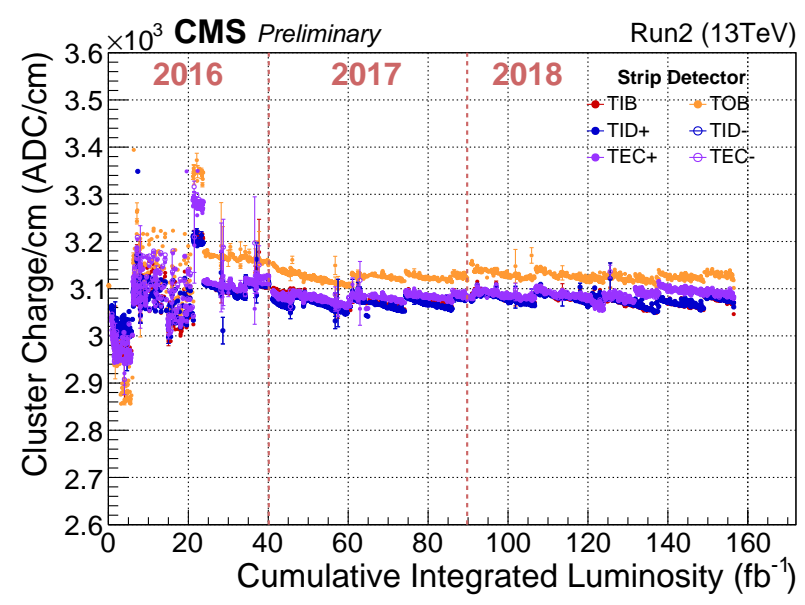

Figure 3: The cluster charge normalized to the traversed silicon length after offline calibration for each partition of the Silicon Strip detector as a function of the delivered LHC integrated luminosity.

One of the main challenges of the LHC Run 2 was a significant increase in the number of overlapping p-p interactions in the detector known as pile-up. Of particular concern for the SST was out-of-time (OOT) pile-up that led to an increase of the strip detector occupancy of about $45 \%$. Since OOT contribution is characterized by a low collected charge, as it is induced by tracks (loopers) that deposit charge out of time with respect to the sampling window, it is suppressed by imposing the requirement on the value of the normalized cluster charge. During local reconstruction, a correction is applied to the strip charge signal in order to guarantee stability over time of the cluster charge most probable value (MPV) in order not to remove in-time hits. The cluster charge normalized to the traversed silicon length after offline calibration for each partition of the Silicon Strip detector as a function of the delivered LHC integrated luminosity is shown in Fig. 3. 
Only clusters used to build a track are considered. The MPV from a Landau fit is shown. Large fluctuations in the cluster charge in the first $20 \mathrm{fb}^{-1}$ of 2016 data are caused by the saturation of the pre-amplifier of the APV chip [4]. The effect had a strong dependence on instantaneous luminosity and resulted in a deviation from a Landau distribution. The effect will be partially mitigated with the updated calibration derived for the Legacy reprocessing of the Run 2 data the CMS collaboration is undertaken. The discontinuities at the later dates in the trend are due to the updates of the analog-opto-hybrid gain and noise/pedestal values.
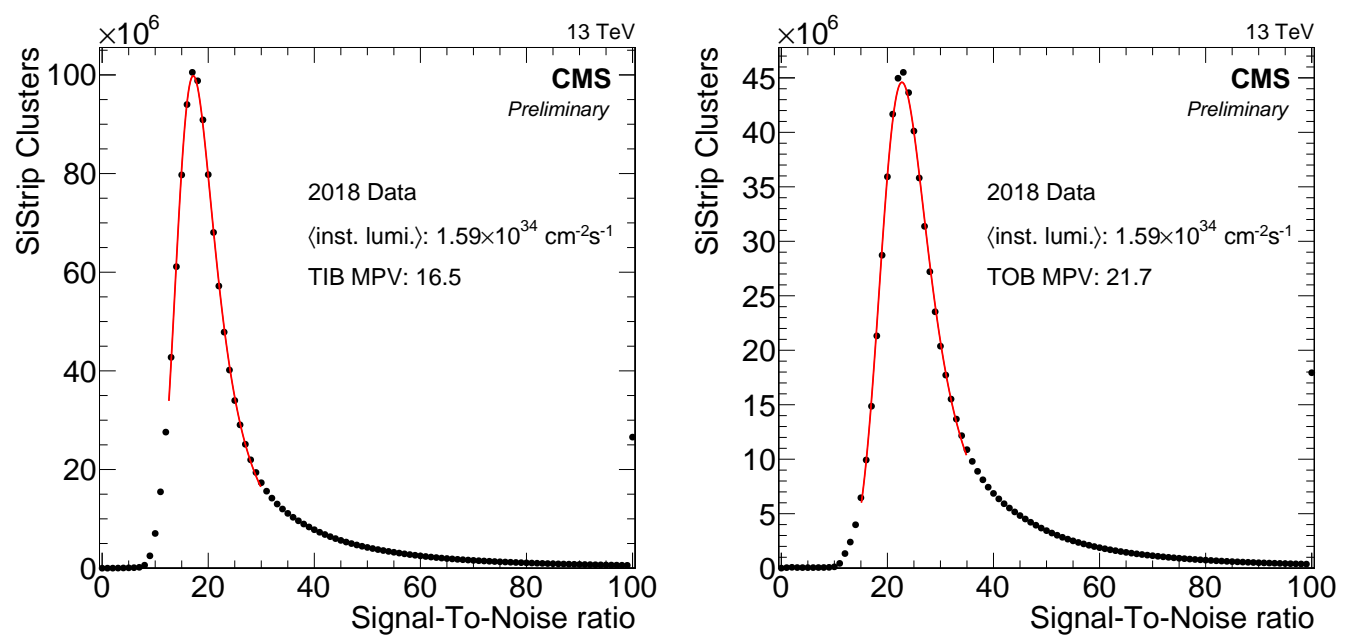

Figure 4: Signal-to-noise ratio for modules in the TIB (left) and TOB (right), corrected for the length of the trajectory inside the silicon sensor to the nominal sensor thicknesses ( 320 or $500 \mu \mathrm{m}$ ). The result of a fit of a Landau distribution convoluted with a Gaussian is shown by the red line.

The signal-to-noise ratio $(\mathrm{S} / \mathrm{N})$ for hits on reconstructed particle trajectories is one of the most important performance characteristics of the silicon strip detectors since it is crucial for the separation of real signals generated by charged particles and fluctuations in the detector noise. Examples of signal-to-noise distributions for TIB and TOB detector partitions are shown in Fig. 4. The distributions are fitted with a Landau convoluted with a Gaussian function. The extracted MPV is used as an estimate of the signal-to-noise ratio. The $\mathrm{S} / \mathrm{N}$ values obtained for the different partitions of the SST during 2018 are summarised in Tab. 1. The signal-to-noise ratio is quite high and amounts to 16-17 for thin and 22 for thick sensors. In Fig. 5, the change of the signal-to-noise ratio measured in p-p collisions during the LHC Run 2 as a function of integrated luminosity is presented. Measurements are performed per partition and split per sensor thickness in the TEC. As expected from irradiation studies, the $\mathrm{S} / \mathrm{N}$ is decreasing with increasing sensor irradiation. At the end of Run $3\left(500 \mathrm{fb}^{-1}\right)$ signal-to-noise ratio of about $12(18)$ is expected in the thin (thick) sensors which will allow collecting high-quality physics data until the end of the detector operational lifetime.

The high signal-to-noise ratio results in excellent in-time hit reconstruction efficiencies for all SST partitions. The hit reconstruction efficiencies as a function of the pile-up are shown in Fig. 6 for four TIB and five TOB layers. As the algorithm is based on inward propagation of the trajectory, no measurement could be performed in the outermost instrumented surfaces (TOB Layer-6 and TEC Disk-9). The hit efficiency decreases with pile-up (and instantaneous luminosity) and are above $98 \%$ at the highest values of pile-up. The observed decrease of the hit efficiencies 
Table 1: Most probable value extracted by fitting a Landau convoluted with a Gaussian function to the $\mathrm{S} / \mathrm{N}$ distribution. Values are shown for different partitions of the Silicon Strip Tracker measured with data recorded in 2018. For TEC, values for thin $(320 \mu \mathrm{m})$ and thick $(500 \mu \mathrm{m})$ modules are given separately.

\begin{tabular}{cccccc}
\hline Detector partition & TIB & TOB & TID & TEC (thin) & TEC (thick) \\
\hline S/N [MPV] & 16.5 & 21.7 & 16.0 & 16.9 & 21.8 \\
\hline
\end{tabular}

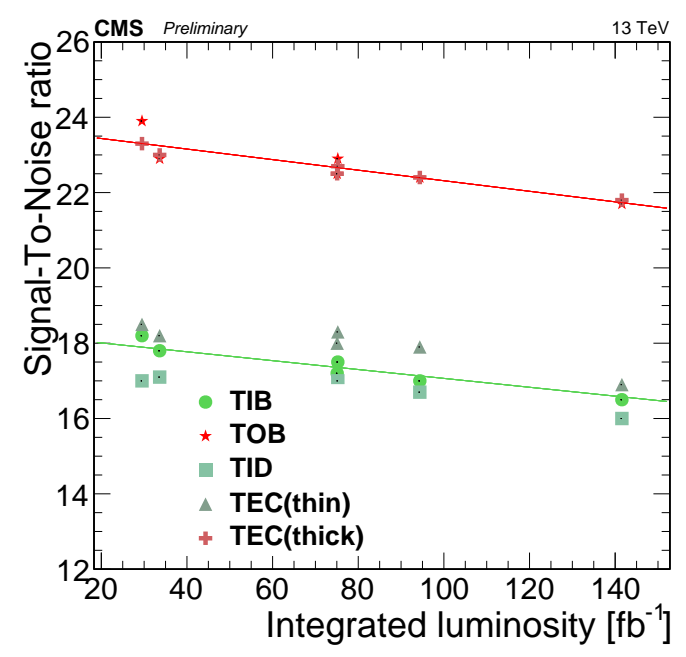

Figure 5: Signal over noise ratio measured in p-p collision during LHC Run 2 as a function of the integrated luminosity.

is attributed to highly ionizing particles (HIP) that release about 100 times larger amount of energy in the strip sensors than a MIP particle. Large energy deposits on only a few strips cause a drop of the APV chip baseline. The recovery can take several beam bunches during which the MIP signal is not large enough to be accepted, leading to efficiency losses. The probability of HIP inducing a saturation of the electronics and consequently a dead-time in the SST was measured in 2018, during a run acquired with the frontend electronics operated in virgin raw mode, i.e. without zero suppression and with a special trigger configuration [5]. The average probability of HIP event occurrence per p-p interaction for all layers of the SST is presented on the left-hand side of Fig. 7, while the parameters of a liner fit performed on the hit efficiency measured as a function of the pileup are shown on the right-hand side. Both measurements display a similar pattern. The predicted hit inefficiencies derived from the average HIP event probabilities agree with the measurements within $20 \%$, thus confirming that HIPs are the main source of hit detection inefficiency.

The spatial resolution of the reconstructed hit positions is measured using the so-called pair method that utilizes tracks with two hits in the same layer, detected in the overlapping regions of the modules [6]. This approach minimizes the effects of multiple scattering and is largely insensitive to misalignment effects. The measured hit resolution for strips with different pitch sizes is compared in Fig. 8 with the expected resolution for binary readout (pitch $/ \sqrt{12}$ ). Independent from the cluster width, values better than the binary resolution are achieved, demonstrating the benefits from charge sharing due to analog readout adopted in the SST. This holds even for the single strip clusters as the 

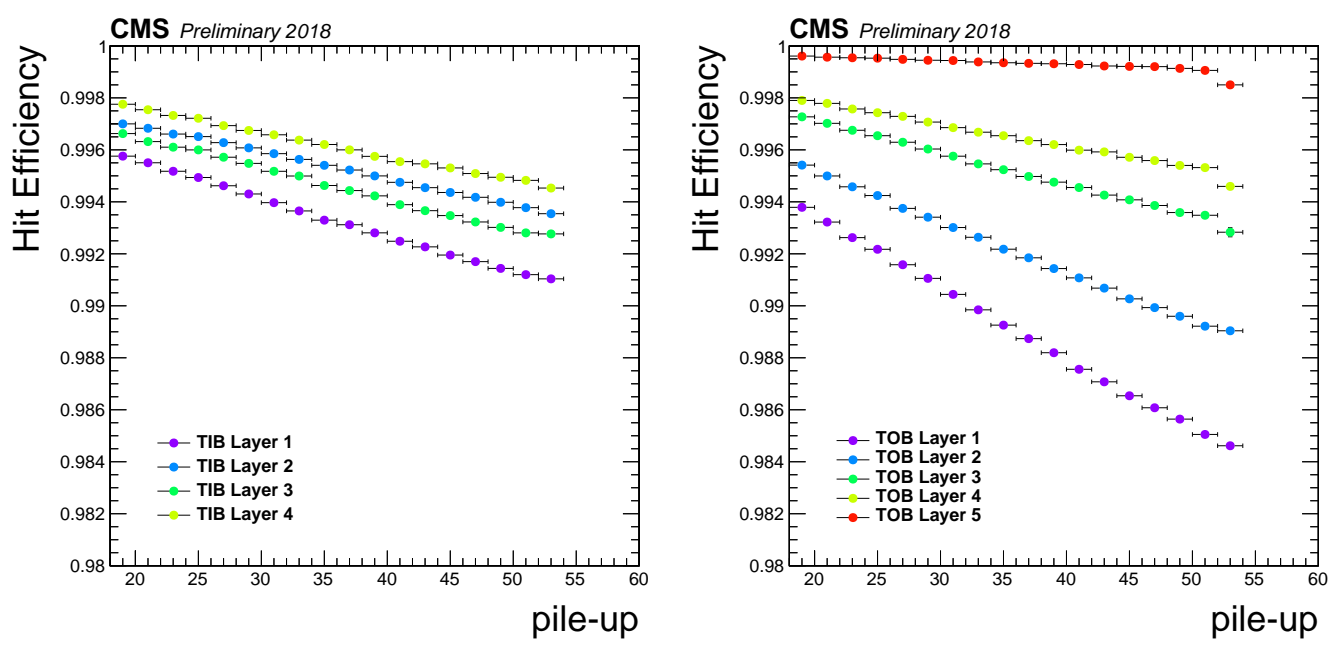

Figure 6: Hit reconstruction efficiency of Silicon Strip detectors for four TIB (left) and five TOB (right) layers as a function of pile-up.
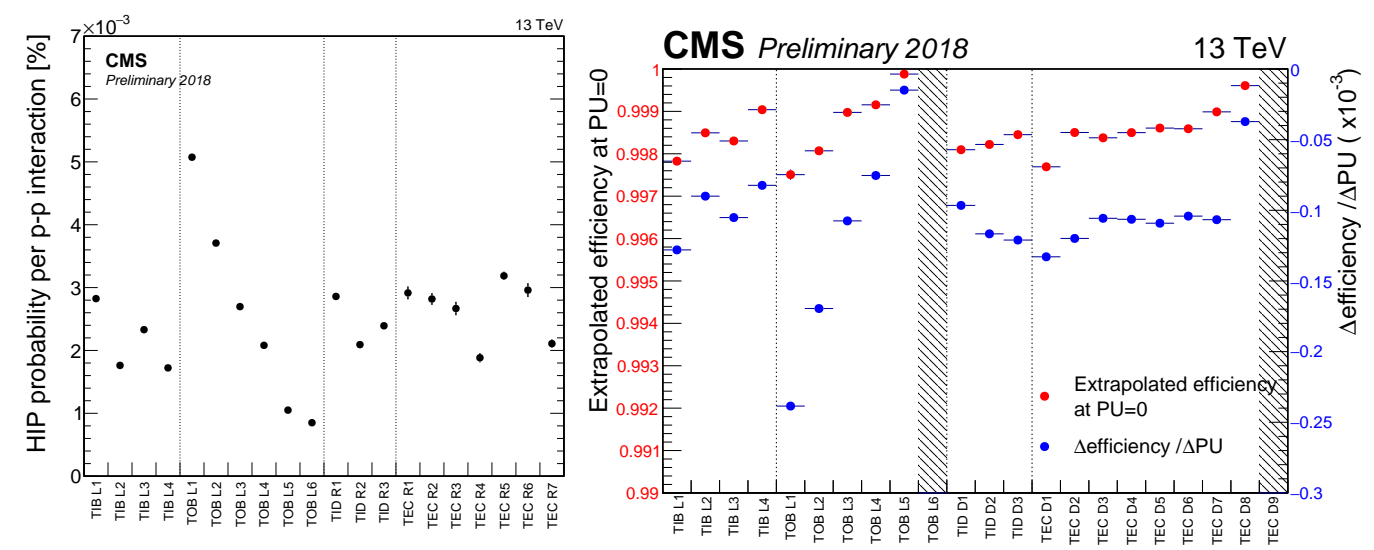

Figure 7: Left: the average probability of HIP event occurrence per p-p interaction for all layers of the Silicon Strip tracker. In the end-cap part, the probability is reported per ring. Right: parameters of a linear fit performed on the hit efficiency measured as a function of the pile-up: the efficiency intercept at zero pile-up (red) and the efficiency vs pile-up slope (blue).

cluster position is corrected based on the detected cluster charge and the local track parameters. In addition, the results show the expected scaling of the measured hit resolution with the strip pitch. The benefits of the cluster sharing are clearly demonstrated in Fig. 9, which shows the improvement of hit resolution for the larger predicted cluster widths defined in [5].

\section{Radiation Effects}

Measurements of the leakage current, full depletion voltages, and the other radiation sensitive variables are performed regularly in order to monitor and assess the impact of the radiation on the SST. 


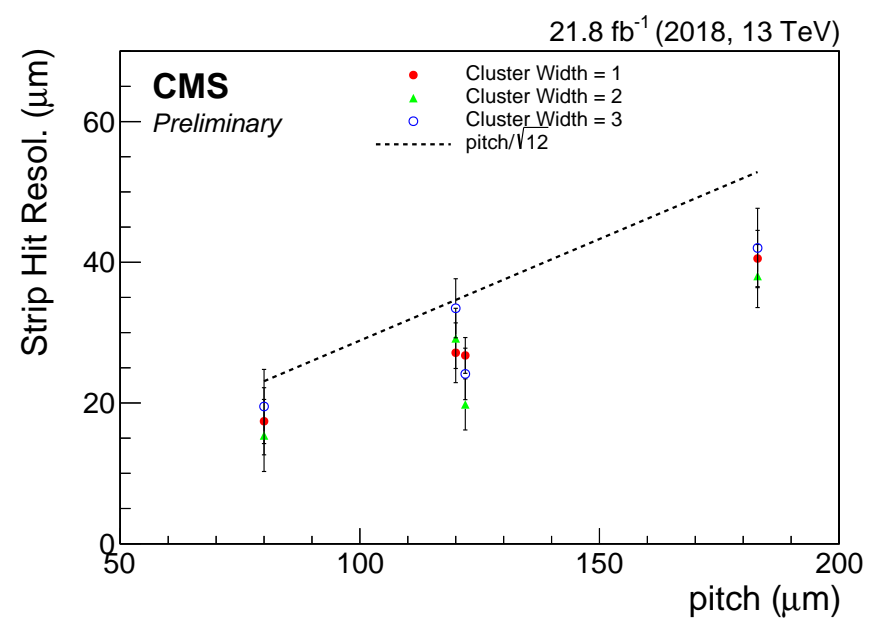

Figure 8: Resolution of the reconstructed hit positions for modules on layers with different strip pitches and for different cluster widths. The expected resolution for binary readout is also shown for comparison.

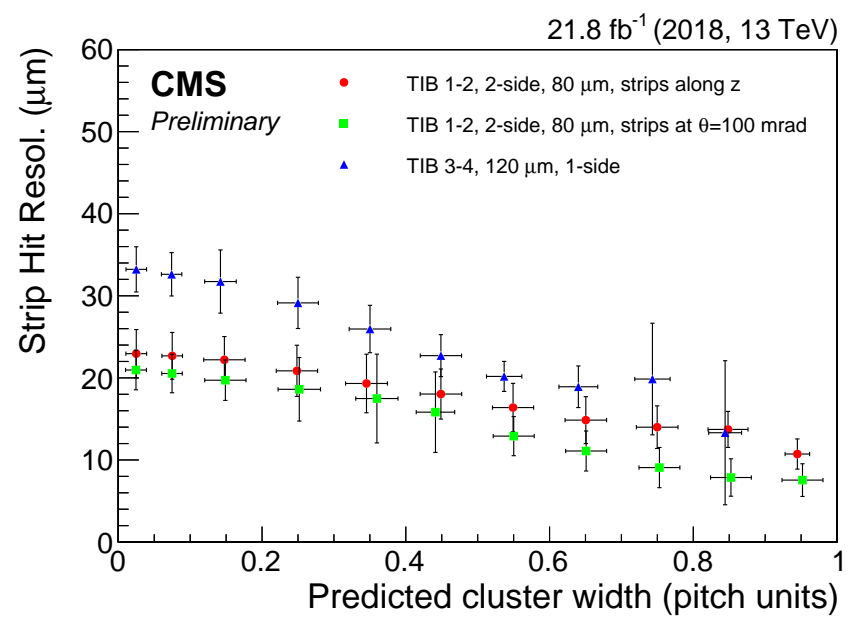

Figure 9: Strip Hit resolution in TIB sub-detector as a function of the expected cluster width, which is defined in [5].

The study of the full depletion voltage $\left(V_{F D}\right)$ evolution is based on periodic bias voltage scans performed during data taking either on the full detector (twice per year) or on a selected group of modules (once per month). The $V_{F D}$ is determined by analyzing the evolution of the cluster width as a function of the applied bias voltage. The value of $V_{F D}$ is extracted from linear fits of the two regimes of the curve as illustrated in Fig. 10 (left). The evolution of the full depletion voltage for TIB Layer 1, obtained by the described method, is presented on the right-hand side of Fig. 10. The model prediction (red line) is superimposed for comparison [8]. The break occurring at integrated luminosity close to $30 \mathrm{fb}^{-1}$ is due to the long shutdown period between LHC Run 1 and 2. The opening of the CMS detector and cooling plant maintenance resulted in extended periods during which the SST was not cooled as well, as it would have been desirable from a radiation damage point of view. This led to a visible accumulation of reverse annealing. Small increases due to the beneficial annealing during winter shutdown periods are visible at around $75 \mathrm{fb}^{-1}$ and 130 

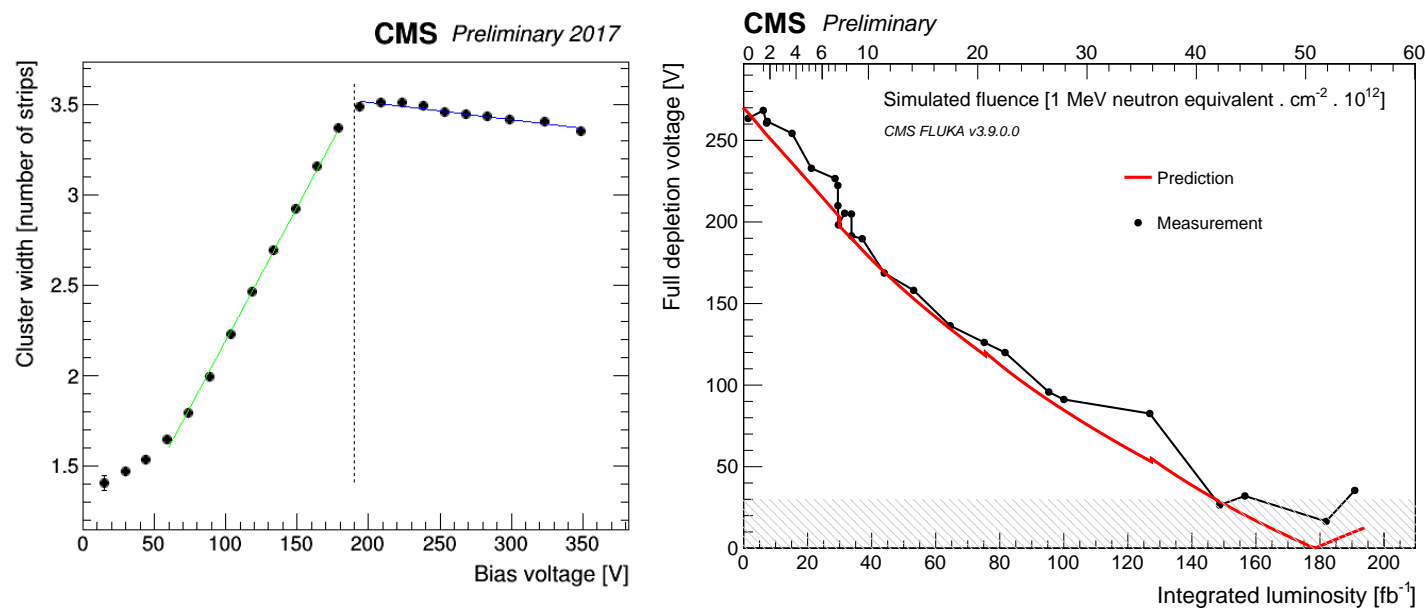

Figure 10: Illustration of the method used to determine the full depletion voltage (left). Evolution of the full depletion voltage for the TIB Layer 1 sensors as a function of the integrated luminosity (right).

$\mathrm{fb}^{-1}$. The shaded area highlights the region at low values of the full depletion voltage where the analysis loses sensitivity. At low values of the bias voltage, the signal-to-noise ratio is too low to gather enough statistics and the increase of the cluster width with the bias voltage is not visible. In such cases, the full depletion voltage is set to the value of the first voltage setting with clusters reconstructed. It is therefore not possible to deduce from the data the exact position of the inversion point. However, the data as well as the model prediction, suggest that the TIB Layer 1 reached the inversion point at the end of LHC Run 2.

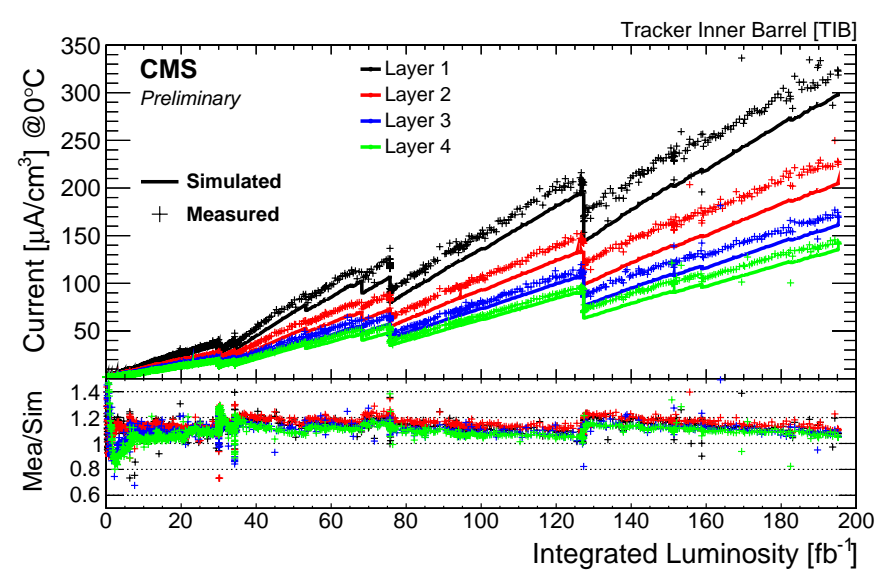

Figure 11: Evolution of the average leakage current for TIB layers as a function of integrated luminosity.

The leakage current is measured by Detector Control Units (DCU), ASICs located on each module and power supply unit [1]. The evolution of the average leakage current per cubic centimeter with luminosity is presented in Fig. 11 for TIB layers. The current is averaged over all sensors (for which data is available) in a given layer, after scaling the current to its equivalent at $0^{\circ} \mathrm{C}$ [7]. The data shown correspond to the whole of LHC Run 1 and Run 2 and were extracted when LHC beam status was stable. The leakage current is simulated using the fluence estimated by 


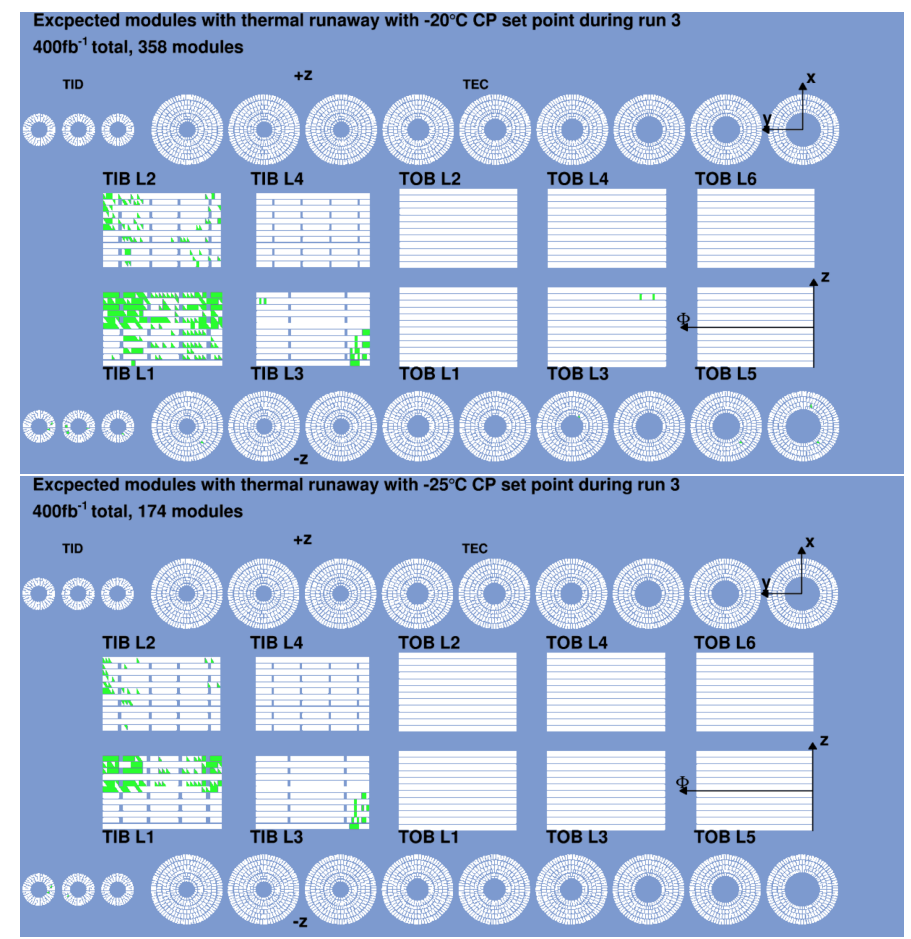

Figure 12: Expected modules with thermal runaway at integrated luminosity of $400 \mathrm{fb}^{-1}$ with coolant temperature set to $-20^{\circ} \mathrm{C}$ (top) and $-25^{\circ} \mathrm{C}$ (bottom).

the FLUKA simulations and the modeled temperature of the tracker. The simulation model parameters are given in [9]. The lower panel shows measured-to-simulated leakage current ratio. These simulations are used to perform projections into the future to estimate the detector's lifetime as well as to optimize annealing to improve the detector performance. While the differences between simulation and measurement as well as the slight difference between Run 1 and 2 require further investigations, the agreement is within the uncertainties of the FLUKA simulations as well as within the uncertainties on the simulation model parameters. Sudden changes in the leakage current are due to the annealing during long shutdowns and end-of-years stops and change of temperature.

In 2017, thermal runaway was observed in several modules in the first layer of TIB subdetector in the regions with degraded cooling contacts or closed cooling loops (passive cooling). The effect was mitigated by decreasing the bias voltage to $200 \mathrm{~V}$ which significantly decreased the leakage current and allow further operation of these modules. For the 2018 data taking, the temperature was lowered from $-15^{\circ} \mathrm{C}$ to $-20^{\circ} \mathrm{C}$ in order not to reach the power supply current limit in the detector regions with passive cooling.

Modules that are expected to experience thermal runaway at the end of Run 3 (total integrated luminosity $400 \mathrm{fb}^{-1}$ ) are shown in Fig 12 , for the coolant temperature set to $-20^{\circ} \mathrm{C}$ (top) and $-25^{\circ} \mathrm{C}$ (bottom). By decreasing the coolant temperature the number of modules experiencing thermal runaway at the end of Run 3 is decreased by a factor of 2 . The effect can also be partially mitigated by switching off the HV in the glued sensors of the stereo modules (TIB 1,2 and TOB $1,2)$. Detailed performance studies are ongoing. Preliminary studies indicate good tracker performance until the end of Run 3. 


\section{Summary and Outlook}

The CMS Silicon Strip Tracker is performing well after ten years of operation. The fraction of active readout channels has been quite stable during the LHC Run 2 and amounts to $\approx 96 \%$ of the total number of channels. Signal-to-noise ratio, hit resolution and hit efficiency are very good and in agreement with the expectations. The predicted radiation effects are visible in many parts of the system. At the beginning of 2018, the operational temperature of the detector was lowered from $-15^{\circ} \mathrm{C}$ to $-20^{\circ} \mathrm{C}$ in order to decrease the leakage current in regions with degraded cooling and prevent thermal runaway. At the end of Run 2, the first layer of the TIB detector reached the inversion point. Changes in the leakage current and depletion voltages are being monitored regularly and compared to the simulations, which are in good shape to model the behavior of the system with increasing irradiation. Simulation studies indicate the occurrence of thermal runaway in the first layers of the TIB subdetector at the end of Run 3 for a coolant temperature of $-20^{\circ} \mathrm{C}$. The number of thermal runaway modules can be reduced by a factor of two by further decreasing the coolant temperature to $-25^{\circ} \mathrm{C}$. The effect can be also partially mitigated by switching off the HV in the glued sensors of the stereo modules (TIB 1,2 and TOB 1,2). The detail performance studies are ongoing. Preliminary studies indicate acceptable tracker performance until the end of Run 3. Further mitigation of the tracking efficiency loss is foreseen to be implemented at the software level.

\section{Acknowledgment}

This work has been partially supported by the Croatian Science Foundation under the project IP-2016-06-3321

\section{References}

[1] CMS Collaboration, The Tracker Project Technical Design Report, 1998, CERN-LHCC-98-06, CMS Collaboration, Addendum to CMS Tracker TDR, 2000, CERN-LHCC-2000-016.

[2] CMS Collaboration, The CMS Experiment at the CERN LHC, 2008, JINST 3 S08004.

[3] G. Cerminara, B. van Besien, Automated workflows for critical time-dependent calibrations at the CMS experiment, J.Phys.Conf.Ser. 664 (2015), 072009, doi:10.1088/1742-6596/664/7/072009

[4] E. Butz, Operation and Performance of the CMS Outer Tracker, PoS Vertex 2017 (2018) 013, doi:10.22323/1.309.0013.

[5] CMS Collaboration, Silicon Strip Tracker Performance results, 2000, CERN-CMS-DP-2018-052.

[6] CMS Tracker Collaboration, Stand-alone Cosmic Muon Reconstruction Before Installation of the CMS Silicon Strip Tracker, JINST 4 (2009) P05004m doi:10.1088/1748-0221/4/05/P05004.

[7] A. Chilingarov, Temperature dependence of the current generated in Si bulk, JINST 8 (2013) P10003, http://dx.doi.org/10.1088/1748-0221/8/10/P10003.

[8] C. Barth, Performance of the CMS Tracker under Irradiation, PhD thesis, IEKP-KA-2013-01, CERN-THESIS-2013-410.

[9] M. Moll, Radiation Damage in Silicon Particle Detectors, PhD thesis, University of Hamburg, 1999. 